\title{
Intermittent 96-Hour Auricular Electroacupuncture for Hot Flashes in Patients with Prostate Cancer: A Pilot Study
}

\author{
Tyvin Rich, MD, FACR, Gerald W. Porter II, MS, ${ }^{1,2}$ Luisel Ricks-Santi, PhD,
}

Tzvi Milshtein, $\mathrm{PE}^{3}$, and Thomas Corbin, $\mathrm{PhD}$, DiplAc ${ }^{4}$

\begin{abstract}
Objective: The hot flash is a common vasomotor disorder that causes distress in menopausal women and that can be debilitating in men with prostate cancer who are treated with androgen deprivation therapy (ADT). The utility of auricular electroacupuncture (AEA) was tested exclusively for a small cohort of men with ADTinduced vasomotor symptoms while the men underwent a course of curative radiotherapy.

Materials and Methods: Prior to and during radiotherapy treatment, men with vasomotor symptoms were given repeated questionnaires regarding severity and frequency of hot flashes, quality of life (QoL), and sleep over a 6-week span of an AEA protocol. Each subject's heart rate variability (HRV) was obtained repeatedly every week in an ambulatory setting with a BlueCardio device (BlueCardio, Miami, FL). The AEA intervention was given with a Neurova device (Nunka Corporation, CM Wellness Clinic, Pompano Beach, FL) that used three needles at Master points Sympathetic, Shen Men, and Point Zero, which were located precisely with a bipolar point finder. Intermittent microcurrent stimulation was given every other week for 96 hours, using a cyclic programmed output of 2 hours on and 2 hours off.

Results: Of 10 men completing the 6-week protocol, all responded with significantly lower frequency, duration, and severity of vasomotor symptoms; QoL and sleep scores improved significantly. The HRV analysis showed significantly lower low-frequency/high-frequency power ratios in each individual, compared to baseline, that were consistent with the subjective responses.

Conclusions: Vasomotor disturbance, caused by gender hormone withdrawal—either naturally or in patients treated with ADT, as in this study - is a well-defined neurophysiologic condition. This disorder is a constellation of findings that reflect autonomic disturbances of excessive sympathetic and reduced parasympathetic activity. AEA intervention with the Neurova device is simple to administer, is well-tolerated, and appears to be effective for restoring autonomic balance. Further evaluation of AEA for vasomotor disturbances could provide more insight into the mechanisms of AEA neuromodulation and potentially lead to approaches for treating not only these symptoms but also other neurologic conditions with components of autonomic disturbances.
\end{abstract}

Keywords: vasomotor symptoms, hot flashes, auricular electroacupuncture, autonomic modulation

\footnotetext{
${ }^{1}$ Hampton University Proton Therapy Institute, Hampton, VA.

${ }^{2}$ Hampton University Cancer Research Center, Hampton, VA.

${ }^{3} \mathrm{CM}$ Wellness Clinic, Pompano Beach, FL.

${ }^{4}$ West Palm Beach Veterans Hospital, West Palm Beach, FL.

(C) Tyvin Rich et al., 2017; Published by Mary Ann Liebert, Inc. This is an Open Access article distributed under the terms of the Creative Commons Attribution License, which permits unrestricted use, distribution, and reproduction in any medium, provided the original work is properly cited.
} 


\section{INTRODUCTION}

$\mathbf{M}$ EN WITH LOCALLY ADVANCED or metastatic prostate cancer are commonly treated with luteinizing hormone-releasing agonists and antiandrogens (androgen deprivation therapy; ADT). ${ }^{1,2}$ Approximately $50 \%$ of men with prostate cancer will receive ADT at some point in their treatment and, in this large population, a major debilitating side-effect can be hot flashes. ${ }^{1,3}$ These hot flashes deteriorate health-related quality of life (QoL) and significantly alter physical function and general health, compared to men not receiving ADT $(P<0.001){ }^{4-6}$ ADT-induced hot flashes are a vasomotor disturbance identical to those in menopausal females, consisting of sweating, an elevated heart rate, a drop in blood pressure (during the attack), and changes in heart rate variability (HRV). ${ }^{7}$ There are also incapacitating alterations in sleep and daily activity and findings of osteoporosis, decreased libido, erectile dysfunction, fatigue, and irritability or depression.

Medical treatments that have been used with limited success are hormone replacement and estrogen receptor modulators (in females), selective serotonin reuptake inhibitors, gabapentin, vitamin E, exercise, deep breathing, relaxation techniques, and lowering ambient room temperature. ${ }^{8,9}$ Intervention trials have shown that there is a $20 \%-$ $30 \%$ placebo effect that can confound the results and that must be recognized when testing new interventions. ${ }^{10}$ This situation merits the exploration of a nonpharmacologic approach with acupuncture for the management of vasomotor symptoms.

The practice of peripheral-point acupuncture for these patients was reviewed extensively in a Cochrane analysis. ${ }^{11}$ Sixteen randomized controlled trials with 1155 women found no evidence that real acupuncture, compared to sham acupuncture, made a difference. Two other well-powered prospective randomized trials, also showed that peripheral acupuncture was no better than a control intervention in women with menopausal hot flashes. ${ }^{12,13}$ In men having ADT treatment during radiotherapy, Ashamala et al. reported that peripheral acupuncture was effective for reducing hot flashes ${ }^{14}$; similar findings were noted in another small study, ${ }^{15}$ in which patients having daily sessions of irradiation made multiple acupuncture interventions quite convenient.

Because of the relatively poor success overall with peripheral-point acupuncture for hot flashes and emerging knowledge of the utility of auricular electroacupuncture (AEA) for modulation of the autonomic nervous system, ${ }^{16}$ the authors chose to evaluate a Neurova device (Nunka Corporation, CM Wellness Clinic, Pompano Beach, FL) that can deliver an intermittent microcurrent to ear points for 96 hours. The purpose of this study was to determine the effect of AEA on hot flashes, QoL, and HRV. This article reports the initial results of a minimally invasive AEA treatment protocol, repeated three times during 6 weeks, in men undergoing fractionated proton beam irradiation plus ADT for locally advanced, nonmetastatic prostate cancer.

\section{MATERIALS AND METHODS}

\section{Patient Population}

The protocol was approved by the Hampton University Proton Therapy Institute (HUPTI), Hampton, VA, institutional review board, and all patients gave written consent conforming to the Helsinki accords for conducting human subject research. Thirty men with locally advanced prostate cancer scheduled for proton beam treatment at HUPTI agreed to be screened for hot flash symptoms. Sixteen patients consented to protocol participation; 10 patients received three courses of intermittent 96-hour AEA per protocol. These men were all diagnosed with localized, advanced prostate cancer, Stages T1c, T2, or T3 disease, with serum prostate-specific antigen levels ranging from 2.3 to $32.8 \mathrm{ng} / \mathrm{mL}$, and Gleason's scores of 7-9. The participants' ages ranged from 54 to 83; all were receiving ADT at the beginning of the protocol.

\section{AEA Protocol}

Patients were treated with AEA for three 96-hour sessions. The treatment was given every other week spanning a total of 6 weeks. The Neurova device is a microcurrentpoint stimulation device. This device is powered by three $1.4-\mathrm{V}$ batteries and produces a $0.1-\mu \mathrm{A}$ current with an impulse interval of $1100 \mathrm{msec}$, and impulse width of $1.1 \mathrm{msec}$ at $1.1 \mathrm{~Hz}$. The stimulation profile is shown in graphic form in Figure 1, and the application to the ear is shown in Figure $2 \mathrm{~A}$ and $\mathrm{B}$. The duration of stimulation was programmed for 2 hours on and then 2 hours off, and cycles for 96 hours. At the end of this stimulation protocol, or when it stopped pulsing because of battery failure, the entire apparatus was easily removed by the subject, or in the clinic office by a nurse, and discarded. After 1 week's rest, the 96hour protocol was repeated on the opposite ear.

The Neurova application was performed by first swabbing the ear gently with an alcohol pad and locating with a digital bipolar point finder, Pointoselect Digital DT (SchwaMedico, Ehringshausen, Germany), the Point Zero, Shen Men, and Sympathetic Master points. ${ }^{17}$ A special forceps was used to grasp the semipermanent needles $(1.0 / 3.0 \mathrm{~mm}$ width $\times 3.1 \mathrm{~mm}$ length needles) that were pushed into these three points. An adhesive mesh stabilized the needles and protected the wired needles from detachment. The Neurova device was activated and connected to an electrogel pad placed behind the ear (Fig. 2). Activity of the device was confirmed before the subject left by the sensation of a slight buzzing at the site of needle insertion.

AEA treatment is very well-tolerated. However, 6 of the 16 patients who had originally consented did not complete 

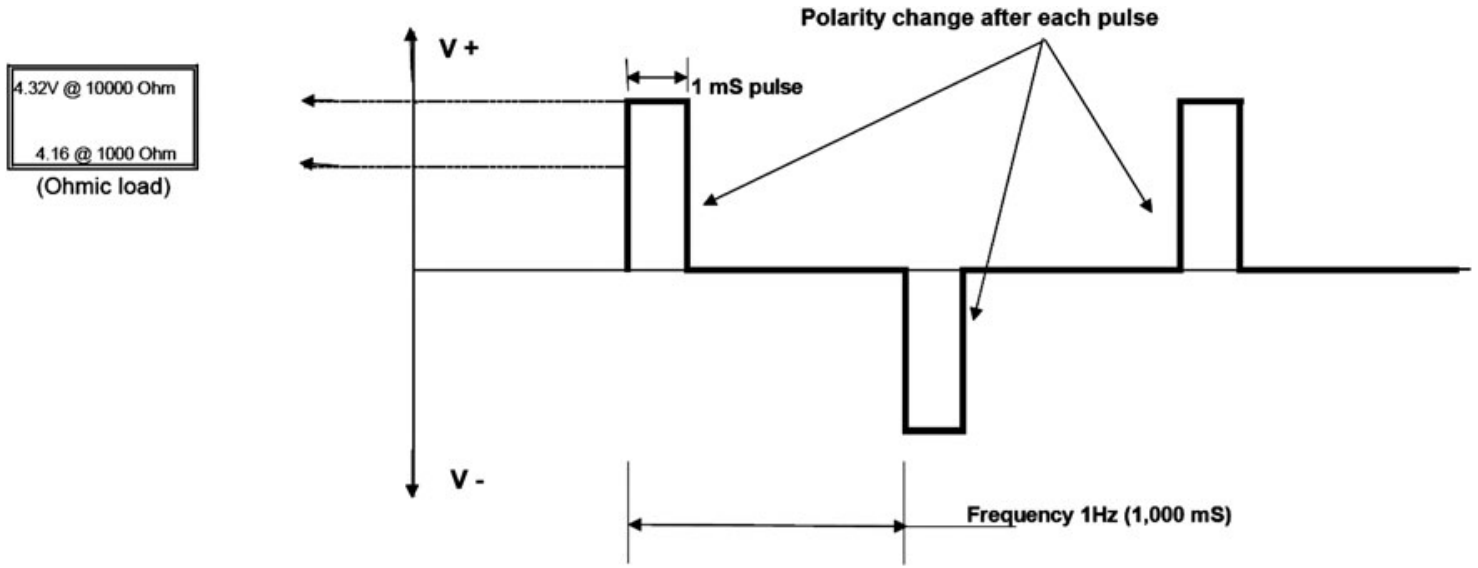

Stimulation Pattern:

The delivered stimulation pattern consists of single pulses with a pulse duration of $1 \mathrm{mS}$

The repetition rate of this pulse is $1 \mathrm{~Hz}$ the current direction is inverted with each pulse

The curve shape is applied for two hours at a time, with an inverted of two hours in between each application. The maximum duration of treatment is $96 \mathrm{Hr}$.

FIG. 1. The stimulation pattern of the Neurova device (Nunka Corporation, CM Wellness Clinic, Pompano Beach, FL) is illustrated giving details of the repetition cycle.

the protocol because: 1 could not tolerate the AEA sensation of microcurrent stimulation; 1 could not tolerate the device during sleep; 1 refused to wear the device because of discomfort caused by lying on the needle insertion sites during sleep; 1 refused to wear the device because of concern about its appearance at the workplace; and 2 did not complete the full protocol for personal reasons.

\section{Questionnaire Administration}

Hot Flash-Related Daily Interference Scale (HFRDIS). ${ }^{18}$ The HFRDIS is a 10 -item scale measuring the degree that hot flashes interfere with nine daily activities; the tenth item measures the degree that hot flashes interfere with overall QoL. The HFRDIS was developed to include daily life activities specific to the impact of hot flashes. Participants rated the degree to which hot flashes have interfered with each item during the previous week using a 0 (did not interfere) to 10 (completely interfered) point scale. A total score was computed by summing items. Higher scores indicated higher interference due to hot flashes, and thus, greater impact on QoL.

Hot flash diary. This diary enabled a detailed, prospective assessment of hot flashes occurring over a 24-hour period. The men were asked to: (1) record the date and time

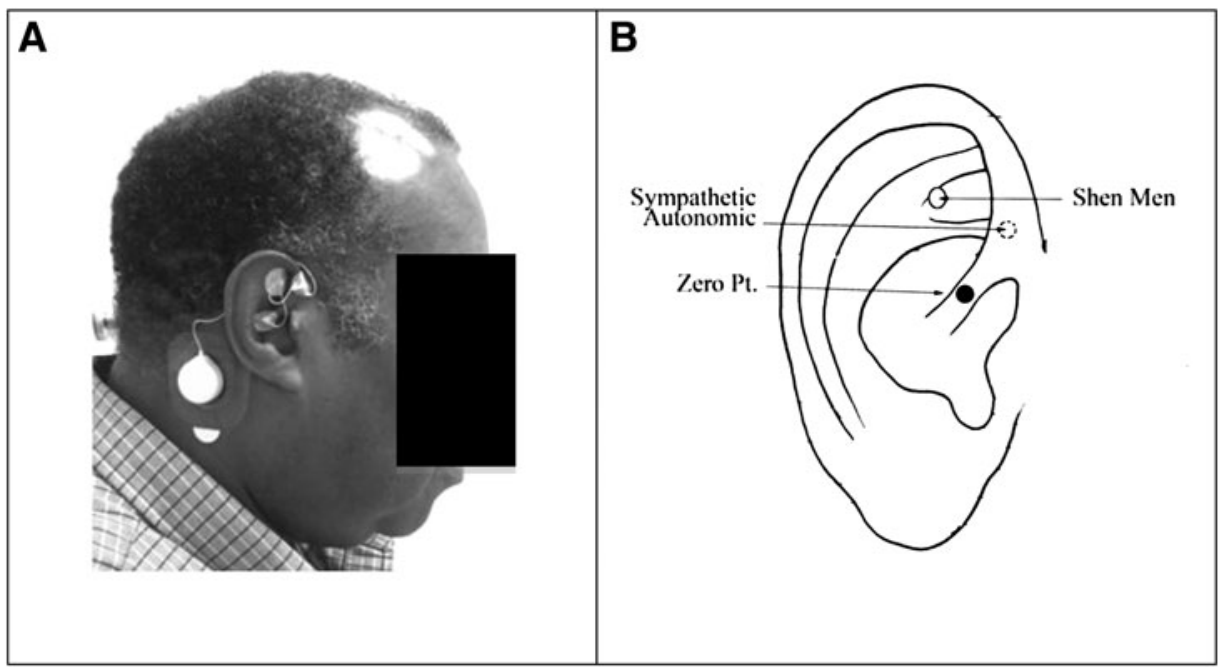

FIG. 2. Placement of the Neurova device (Nunka Corporation, CM Wellness Clinic, Pompano Beach, FL) for auricular electroacupuncture. (A) Device on patient. (B) Auricular acupuncture points. 
of each hot flash; (2) rate the severity of each hot flash using a 0 (not at all) to 10 (extreme) Numeric Rating Scale; and (3) record the duration of each hot flash in minutes. Hot flash frequencies (HFFreq) were calculated as the number of hot flashes recorded per day. Mean severity ratings were calculated by summing individual severity ratings and dividing by the number of hot flashes experienced. Mean duration ratings were calculated similarly. Frequency counts and mean severity and duration ratings were calculated separately.

Expanded Prostate Cancer Index Composite (EPIC). ${ }^{19}$ EPIC is a robust prostate cancer QoL instrument that complements prior instruments by measuring a broad spectrum of urinary, bowel, sexual, and hormonal symptoms, thereby serving as a unique tool for comprehensive assessment of QoL issues that are important in contemporary prostate cancer management. Higher scores meant poorer QoL.

General Sleep Disturbance Scale. ${ }^{20}$ The GSDS is a 21-item scale initially designed to evaluate the incidence and nature of sleep disturbances in employed women. Questions pertained to a variety of general sleep issues, including: problems initiating sleep; waking up during sleep; waking too early from sleep; quality of sleep; quantity of sleep; fatigue and alertness at work; and the use of substances to induce sleep. Higher scores meant poorer sleep.

\section{HRV Evaluation}

All patients were instructed in use of a BlueCardio device (BlueCardio, Miami, FL) to collect heart rate data. This device is a credit-card-sized monitor that weighs $38 \mathrm{~g}$, measures
$4.5 \mathrm{~cm} \times 8.5 \mathrm{~cm} \times 0.7 \mathrm{~cm}$, and is held between the fingers for 300 seconds. The heart rate data are then sent by Bluetooth $^{\circledR}$ connection to a smartphone application that uploads over the internet to a HIPPA [Health Insurance Portability and Accountability Act]-compliant server, where the data are archived and analyzed. The analysis provided HRV functions of time and frequency domain variables using Kubios software (Kubios, Kuopio, Finland). Lower lowfrequency-to-high-frequency (LF/HF) ratios indicated autonomic balance.

\section{Statistics}

The questionnaire data were analyzed with SPSS software (version 22.0, IBM, Armonk, NY). Analysis of variance was utilized to measure differences in hot flash frequency, QoL, and HRV over the 6-week duration of the study. A $P$-value of 0.05 was considered to be statistically significant.

\section{RESULTS}

The results showed that there was an incremental reduction of symptoms over the 6 weeks and, overall, there were significant improvements in all patient responses at the end of 6 weeks (Table 1). The EPIC scores were collected at baseline (week 0 and week 6). The general sleepdisturbance scale scores were collected at baseline, week 3 , and week 6. The EPIC and general sleep distress scores were reduced significantly at 6 weeks $(P<0.0001$ and $P<0.05$, respectively; Table 1 and Fig. 3; see EPIC and GSDS graphs). The QoL, HFFreq, and hot flash duration and intensity scores were collected at baseline and weeks 1

Table 1. Descriptive Statistics of Each Questionnaire for Each Week of the AEA Protocol

\begin{tabular}{|c|c|c|c|c|c|c|}
\hline Week & Parameters & $E P I C$ & $Q o L$ & HFRDIS & HFFreq & GSDS \\
\hline \multirow[t]{2}{*}{ Baseline } & Mean & 3.9 & 5.2 & 35.8 & 77.7 & 62.9 \\
\hline & SD & 0.316 & 1.814 & 17.197 & 30.681 & 5.84 \\
\hline \multirow[t]{2}{*}{ Week 1} & Mean & - & 5.1 & 31.8 & 63.3 & - \\
\hline & SD & - & 1.663 & 12.191 & 19.732 & - \\
\hline \multirow[t]{2}{*}{ Week 2} & Mean & - & 6.1 & 47.2 & 75.8 & - \\
\hline & SD & - & 1.287 & 23.055 & 21.358 & - \\
\hline \multirow[t]{2}{*}{ Week 3} & Mean & - & 4.1 & 30.9 & 43.8 & 57.3 \\
\hline & $\mathrm{SD}$ & - & 2.132 & 17.773 & 17.925 & 7.499 \\
\hline \multirow[t]{2}{*}{ Week 4} & Mean & - & 4.5 & 33.7 & 45.1 & - \\
\hline & SD & - & 1.716 & 11.006 & 26.48 & - \\
\hline \multirow[t]{2}{*}{ Week 5} & Mean & - & 3.1 & 21.6 & 19.6 & - \\
\hline & $\mathrm{SD}$ & - & 1.287 & 10.48 & 13.117 & - \\
\hline \multirow[t]{3}{*}{ Week 6} & Mean & 1.7 & 2.9 & 20.5 & 21 & 37 \\
\hline & $\mathrm{SD}$ & 0.949 & 1.449 & 10.352 & 15.825 & 10.791 \\
\hline & Significance & $P<0.0001$ & $P<0.0001$ & 0.005 & $P<0.0001$ & $P<0.0001$ \\
\hline
\end{tabular}

AEA, auricular electroacupuncture; SD, standard deviation; EPIC, Expanded Prostate Cancer Index Composite; QoL, Quality of Life; HFRDIS, Hot Flash-Related Daily Interference Scale; HFFreq, Hot Flash Frequency; GSDS, General Sleep Disturbance Scale. 


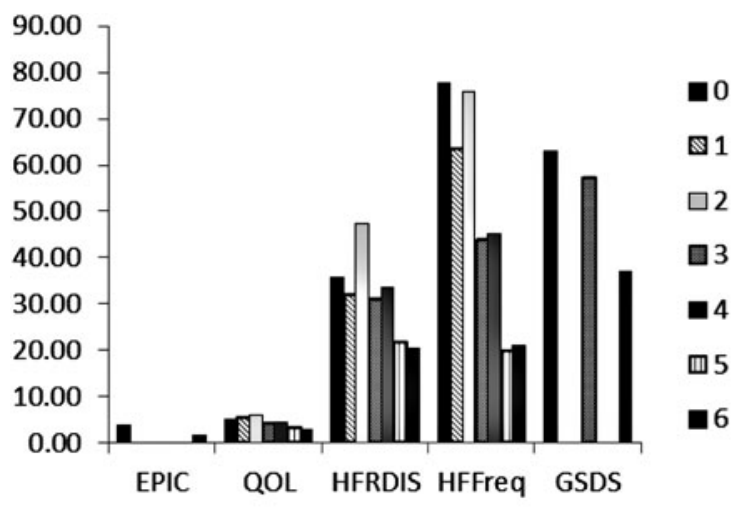

QOL

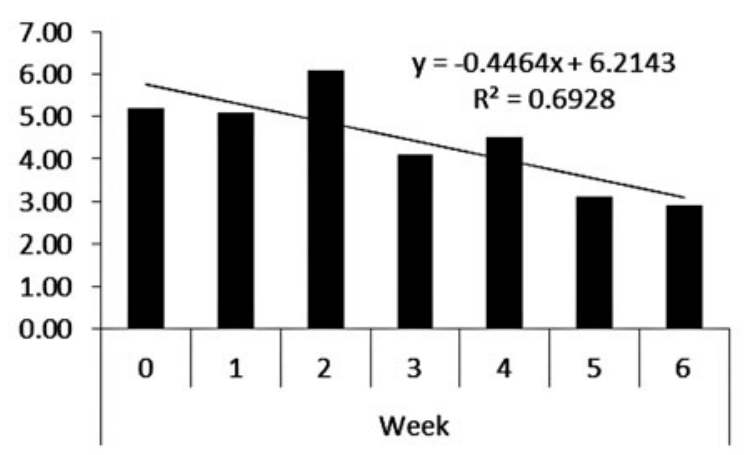

HFFreq

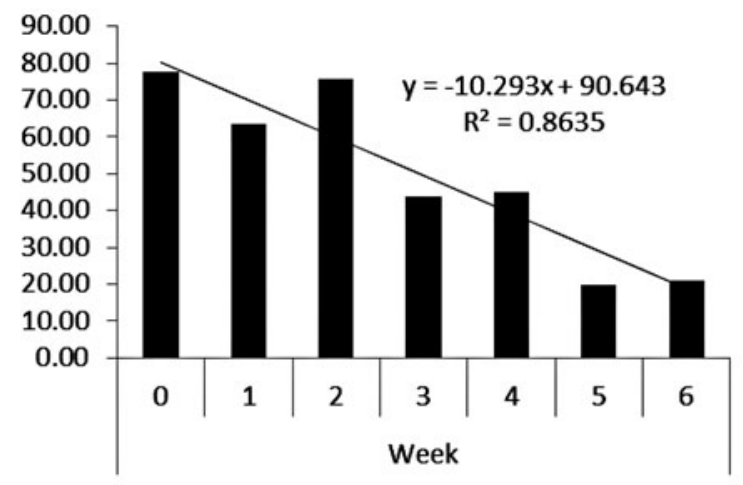

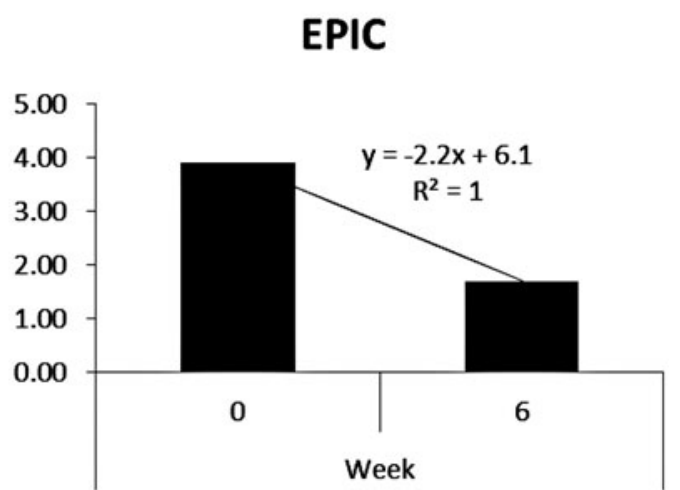

\section{HFRDIS}

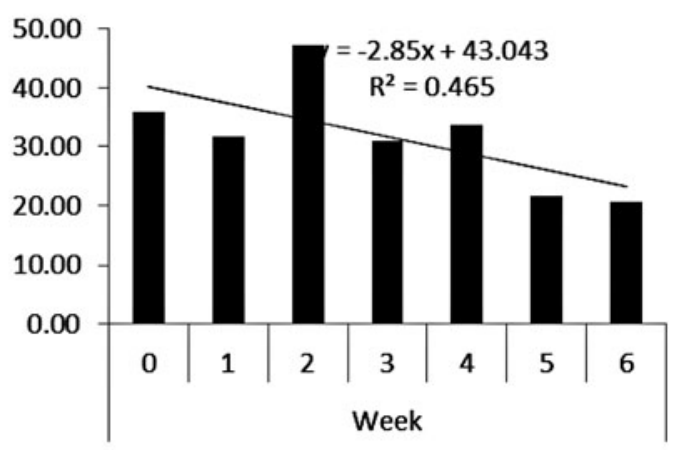

GSDS

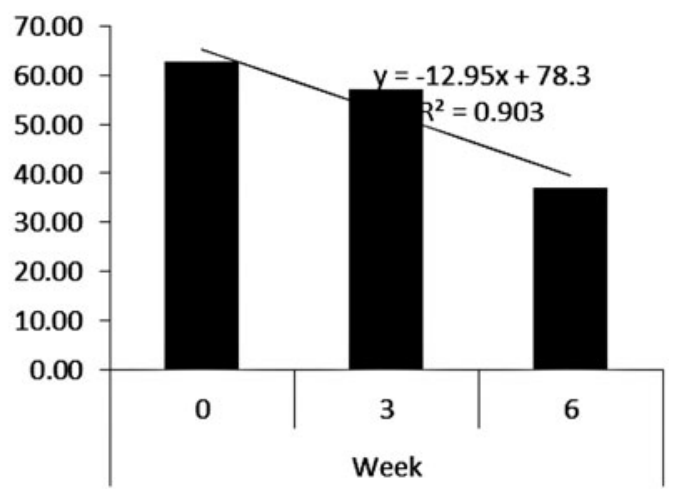

FIG. 3. Summary scores for Expanded Prostate Cancer Index Composite (EPIC), Quality of Life (QoL), Hot Flash-Related Daily Interference Scale (HFRDIS), Hot Flash Frequency (HFFreq), and General Sleep Disturbance Scale (GSDS) by week (see text for details).

through 6. All of the aforementioned scores were reduced significantly after AEA treatment $(P<0.05$ for all scores; Table 1 and Fig. 3; see graphs for QoL, HFRDIS, and HFFreq).

Table 2 shows the weekly LF/HF power ratios calculated by the BlueCardio program. Statistical analysis revealed progressively lower LF/HF ratios for each patient by the end of the AEA protocol $(P<0.0001)$. Figure 4 shows how the scores fluctuated for each patient and the trends of decreasing scores for each patient.

\section{DISCUSSION}

In all patients, the frequency and severity of hot flashes improved significantly with AEA intervention for 6 weeks, 
Table 2. HRV Low-Frequency to High-Frequency Power Ratios by Patient for Each Week of the AEA Protocol ${ }^{a}$

\begin{tabular}{|c|c|c|c|c|c|c|c|}
\hline Patient & Baseline & Week 1 & Week 2 & Week 3 & Week 4 & Week 5 & Week 6 \\
\hline 1 & 2.18 & 17.7 & 6.09 & 3.02 & 4.88 & 2.78 & 1 \\
\hline 2 & 2.24 & 9.4 & 5.89 & 4.75 & 2.6 & 1.69 & 0 \\
\hline 3 & 2.28 & 2.42 & 1.68 & 2.35 & 1.46 & 0.846 & 0.431 \\
\hline 4 & 3.19 & 9.8 & 3.67 & 2.45 & 3.01 & 1.09 & 1.1 \\
\hline 5 & 3.83 & 2.67 & 5.1 & 1.66 & 1.13 & 0.607 & 0.521 \\
\hline 6 & 3.97 & 3.2 & 4.07 & 0.537 & 0.933 & 0.492 & 1.26 \\
\hline 7 & 3.97 & 6.36 & 1.18 & 0.281 & 1.51 & 0.736 & 1.41 \\
\hline 8 & 5.69 & 0.677 & 1.66 & 0.677 & 0.281 & 0.072 & 0.55 \\
\hline 9 & 6.88 & 6.58 & 2.78 & 1.06 & 3.24 & 1.03 & 2.24 \\
\hline 10 & 12.4 & 11.5 & 9.25 & 3.03 & 4.88 & 1.48 & 3.01 \\
\hline
\end{tabular}

${ }^{a}$ Although there was variation of the low-frequency/high-frequency (LF/HF) ratios from week to week, by the end of the AEA protocol assessed at week 6, all patients had reduced ratios. The LF/HF power ratios were calculated from the data collected by repeated weekly measurements using the BlueCardio (BlueCardio, Miami, FL) device and analyzed with Kubios software (Kubios, Kuopio, Finland). Statistical analysis showed that the mean LF/ HF ratios for the entire group was reduced from baseline, compared to week $6(\mathrm{p}<0.0001)$.

HRV, heart rate variability; AEA, auricular electroacupuncture.

compared to each individual's baseline. There are also improvements in somatic symptom dimensions of the EPIC and QoL questionnaires, and sleep scores on the GSDS $(P<0.05)$. Although part of this could represent a placebo effect-an important known confounder in the literature on hot flashes-the current study showed that all patients had objective reductions of HRV LF/HF ratios, reflecting evolution toward stable autonomic nervous system (ANS) performance with AEA. The sympathovagal imbalance-a proposed core neurophysiologic mechanism of autonomic disruption driving vasomotor symptoms-was corrected with this nonpharmacologic intervention. The duration of treatment for these patients was limited by the protocol, but there is no reason that longer AEA could not be used as needed for symptomatic patients.

The withdrawal of gender hormones that causes vasomotor symptoms is postulated to work through hypothalamic $\alpha-2$ adrenergic receptors and increased brain norepinephrine levels. ${ }^{21}$ This creates a narrow thermoneutral zone where small increases in body temperature result in a "confused" hypothalamus sending a top/down signal to the ANS network, causing sympathetic activation triggering vasomotor symptoms. One predominant symptom is well-characterized by altered galvanic skin resistance measurements indicating excitation of the sweat glands by sympathetic cholinergic fibers. There are also associated findings of tachycardia, vasodilation, and lower blood pressure during the event. Other supporting data for increased sympathetic drive are the elevation of the norepinephrine metabolite, 3-methoxy-4-hydroxyphenylglycol that is increased in plasma of patients with hot flashes, and pharmacologic interventions with yohimbine, an $\alpha 2$ adrenergic antagonist, and with clonidine (a $\alpha 2$-adrenergic agonist) that affect hot flashes. ${ }^{21}$

The present authors' HRV analysis and several other researchers have found changes in HRV, indicating that vasomotor disturbances can involve both sides of the ANS. $^{22}$ On the one hand, elevated low-frequency HRV during a 5-minute block at the onset of a nocturnal physiologic hot flash compared to a 5-minute block after a hot flash, regardless of sleep or wake state, is consistent with sympathetic activation. ${ }^{23}$ However, other data show decreased high frequency power (HF) occurring during vasomotor disturbances, indicating a picture of both reduced vagal activity (where the "brakes" are missing) and increased sympathetic activity, like the pressing of the accelerator in the automobile analogy. ${ }^{24}$ Reasons for these discrepancies of HRV measurements between studies could, in part, be due to differences in methodology, with hot flashes being: triggered by a stressor or spontaneously occurring; physiologically or subjectively assessed; recorded during different time periods (day and/or night). There could also be differences in the definitions of hot flashes and HRV frequency bands. ${ }^{22-24}$

It is well-known that changes in HRV measurements are highly sensitive to changes in sleep-wake states, arousals from sleep, mental stressors, or emotional states that are associated with substantial changes in autonomic regulation. Isolation of the vasomotor event from mental arousal (and physically introduced artifacts) can be accomplished by monitoring HRV during sleep in subjects. HF HRV loss is found exclusively during spontaneously occurring vasomotor disturbances during non-rapid eye movement sleep in perimenopausal women. ${ }^{25}$ Although the present study's positive results were obtained with a 96-hour AEA protocol, one implication from the HRV observations made at night is that there might be utility in monitoring for vasomotor disturbance during the rest/sleep phase; it would be helpful to design interventions for when clear-cut evidence of the vasomotor event is happening.

The present study showed that vasomotor disturbance can be corrected nonpharmacologically by auricular electroacupuncture 
A

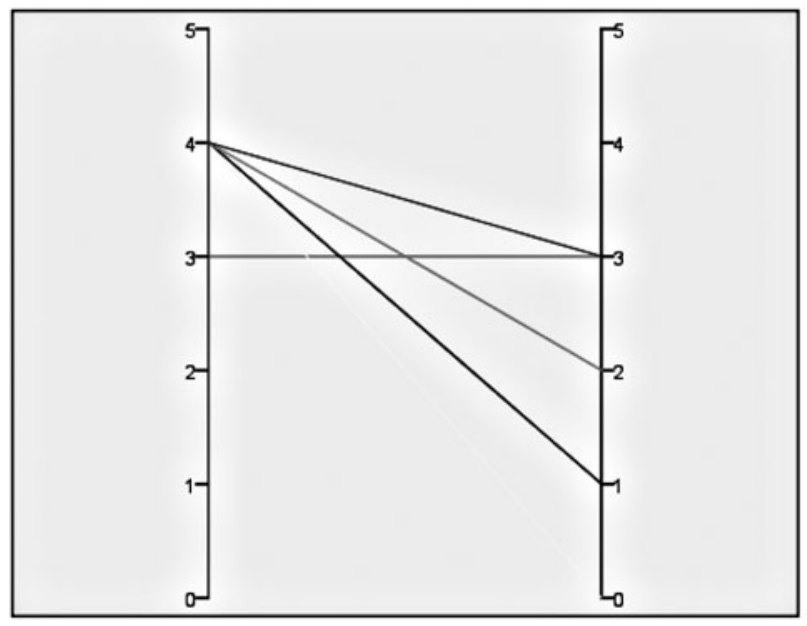

C

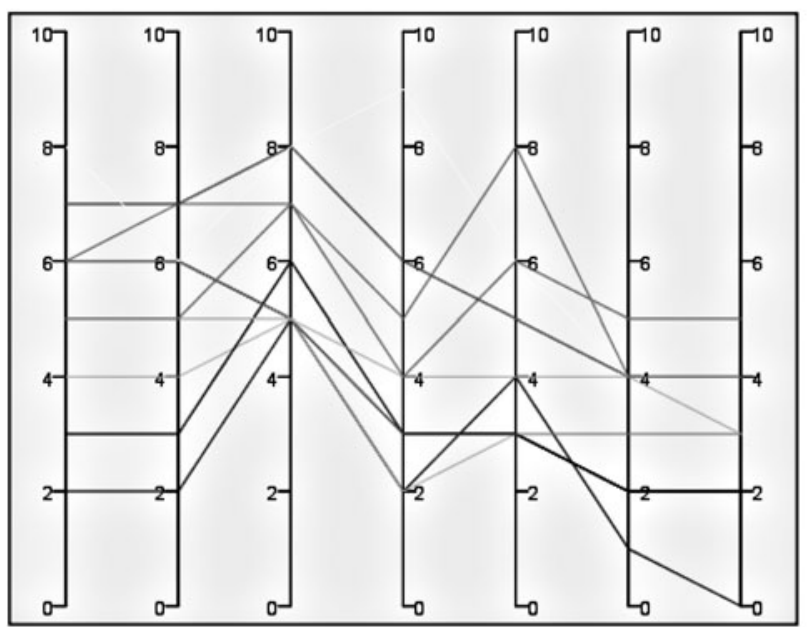

B

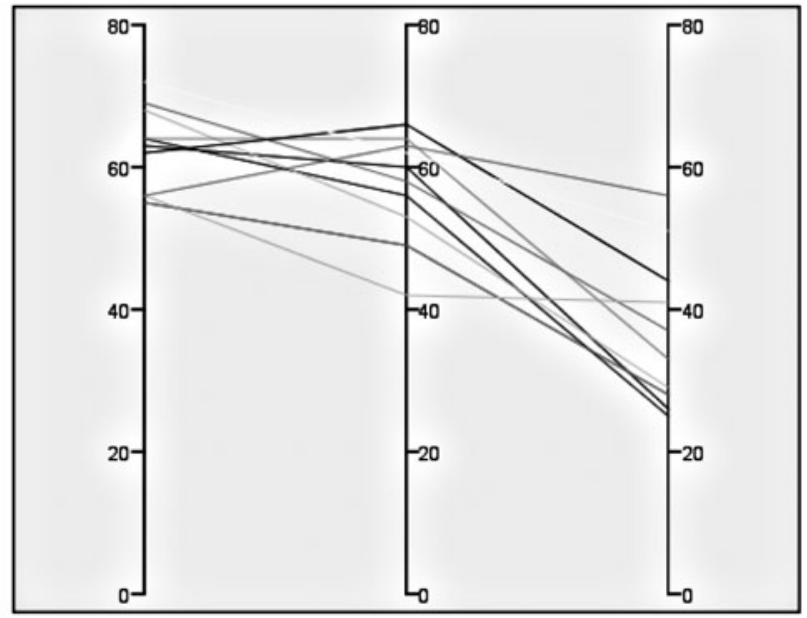

D

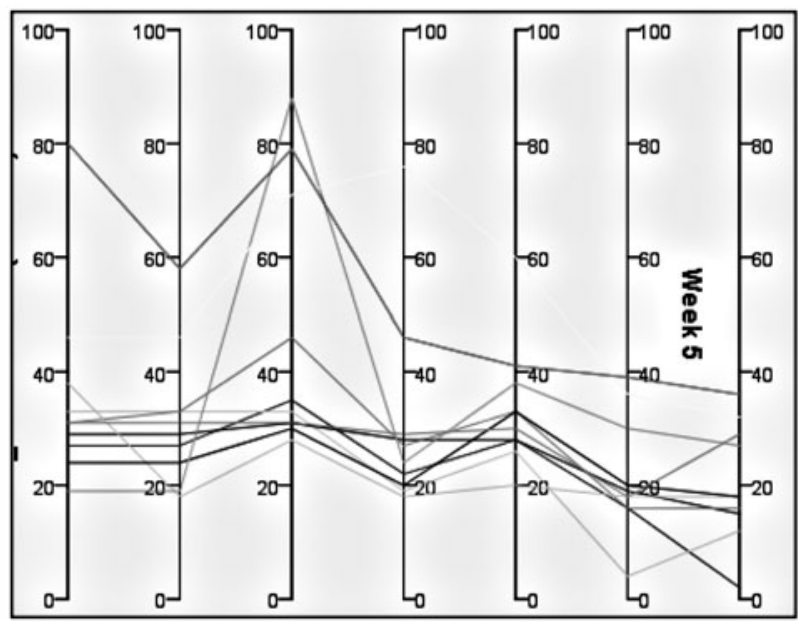

E

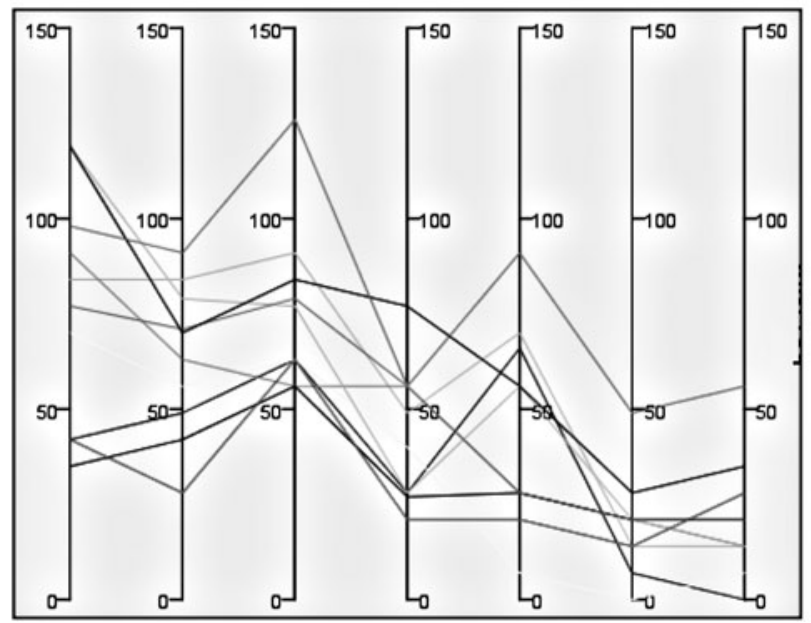

FIG. 4. Scores for each patient by week for (A) Quality of Life (QoL; baseline and week 6), (B) General Sleep Disturbance Scale (GSDS; baseline, week 3, and week 6), (C) Expanded Prostate Cancer Index Composite (EPIC; baseline to week 6), (D) Hot FlashRelated Daily Interference Scale (HFRDIS), and (E) Hot Flash Frequency (HFFreq). 
through what is likely some level of autonomic modulation. The Master points chosen for the present study's protocol are thought to activate both the parasympathetic and sympathetic ANS network. This has been confirmed in humans directly, with transcutaneous stimulation to the cymba concha showing that functional magnetic resonance imaging detected activation of classic vagal projections, while the earlobe activated the spinalthalamic nucleus. ${ }^{26}$ These imaging measurements confirmed the notion that auricular stimulation results in broad signaling (modulation) of the ANS. Other auricular stimulation studies for postoperative pain $^{27}$ and depression $^{28}$ have been associated with changes in HRV consistent with the present study's findings. In particular, AEA at the Shen Men and Point Zero ear points resulted in increased HF-HRV (parasympathetic) activity. ${ }^{27}$

Another dimension of the present study is the issue of why 96-hour intermittent AEA might be superior to peripheral acupuncture intervention. This argument may not only relate to the mechanism of neuromodulation but to the plain fact that the present study's protocol delivered a more-therapeutic "dose" based on consideration of a "neural stimulation $\mathrm{X}$ time" model. The Neurova treatment algorithm used in this protocol delivered 48 hours of neuromodulation per week, and a total of 144 hours of AEA over 6 weeks, compared to only $\sim 4.5$ hours of peripheral acupuncture when administered for 6 weekly sessions of 45 minutes. From a practical perspective, the time it takes to apply the Neurova device is 15 to 20 minutes for each session (totaling $<1$ hour overall for the present authors' entire protocol) to locate the points, insert three needles, and activate the device, compares favorably to tying up the patient, the doctor, and nursing staff in a clinic room for a total of $4.5+$ hours to perform conventional acupuncture six times over a typical course of radiotherapy.

A limitation of this study was the small number of patients. The present authors are continuing to accrue more patients to conduct a modified study wherein hemodynamic and galvanic skin measurements will be made to describe precisely and quantify the vasomotor attack. Another limitation was the lack of a control group; that concern can be addressed in a future study. The current authors are awaiting approval of National Institutes of Health funding for further studies in which a randomized controlled study of AEA versus a sham intervention could be conducted.

However, the design of the current trial, in which each patient's data were compared to each patient's own baseline, plus the addition of objective HRV data, strongly support the study's initial conclusions. Further studies to validate the AEA approach for vasomotor symptoms are warranted.

\section{CONCLUSIONS}

The vasomotor symptoms occurring with hot flashes are a debilitating human neurophysiologic disorder. Monitoring of vasomotor symptoms is feasible with HRV that can be incorporated into clinical studies of neuromodulation of the ANS with AEA.

\section{ACKNOWLEDGMENTS}

The authors wish to thank the supportive HUPTI nursing staff: Cynta Lytle, RN; Tiffany Minor, RN; Diamora Fernandez, RN; Allan Thornton, MD; and Christopher Sinesi, MD; as well as undergraduate Hampton University students Tyanna Jones-Gray and Nina Wyatt for collating the data forms. The authors also extend thanks for financial support from the Virginia Department of Health and from Ronald Maxson.

\section{AUTHOR DISCLOSURE STATEMENT}

The authors have no financial disclosures.

\section{REFERENCES}

1. Shahinian VB, Kuo YF, Freeman JL, Orihuela E, Goodwin JS. Increasing use of gonadotropin-releasing hormone agonists for the treatment of localized prostate carcinoma. Cancer. 2005;103(8):1615-1624.

2. Kaisary AV. Evaluating the use of early hormonal therapy in patients with localised or locally advanced prostate cancer. Prostate Cancer Prostatic Dis. 2005;8(2):140-151.

3. Meng MV, Grossfeld GD, Sadetsky N, Mehta SS, Lubeck DP, Carroll PR. Contemporary patterns of androgen deprivation therapy use for newly diagnosed prostate cancer. Urology. 2002;60(3[suppl1]):7-11;discussion:11-12.

4. Lubeck DP, Grossfeld GD, Carroll PR. The effect of androgen deprivation therapy on health-related quality of life in men with prostate cancer. Urology. 2001;58(2[suppl1]): 94-100.

5. Dacal K, Sereika SM, Greenspan SL. Quality of life in prostate cancer patients taking androgen deprivation therapy. J Am Geriatr Soc. 2006;54(1):85-90.

6. Talcott JA. New era in prostate-cancer care: What we know and what we don't. Lancet Oncol. 2003;4(4):204-205.

7. Loprinzi CL, Barton DL, Rhodes D. Management of hot flashes in breast-cancer survivors. Lancet Oncol. 2001;2(4): 199-204.

8. Loprinzi L, Barton DL, Sloan JA, et. al. Pilot evaluation of gabapentin for treating hot flashes. Mayo Clin Proc. 2002; 77(11):1159-1163.

9. Shanafelt TD, Barton DL, Adjei AA, Loprinzi CL. Pathophysiology and treatment of hot flashes. Mayo Clin Proc. 2002; 77(11):1207-1218.

10. Sloan JA, Loprinzi CL, Novotny PJ, Barton DL, Lavasseur BI, Windschitl H. Methodologic lessons learned from hot flash studies. J Clin Oncol. 2001;19(23):4280-4290. 
11. Dodin S, Blanchet C, Marc I, et. al. Acupuncture for menopausal hot flushes. Cochrane Database Syst Rev. 2013;7: CD007410.

12. Vincent A, Barton DL, Mandrekar JN, et al. Acupuncture for hot flashes: A randomized, sham-controlled clinical study. Menopause. 2007;14(1):45-52.

13. Ee C, Xue C, Chondros P, Myers SP, French SD, Teede H, Pirotta M. Acupuncture for menopausal hot flashes: A randomized trial. Ann Intern Med. 2016;164(3):146-154.

14. Ashamalla H, Jiang ML, Guirguis A, Peluso F, Ashamalla M. Acupuncture for the alleviation of hot flashes in men treated with androgen ablation therapy. Int J Radiat Oncol Biol Phys. 2011;79(5):1358-1363.

15. Hirsch LM, Goldstein LE. Acupuncture for hot flashes in men treated with androgen deprivation therapy. Can J Urol. 2015; 22(4):7938-7941.

16. da Silva MAH, Dorsher PT. Neuroanatomic and clinical correspondences: Acupuncture and vagus nerve stimulation. $J$ Altern Complement Med. 2014;20(4):233-240.

17. Oleson T. Auriculotherapy Manual Chinese and Western Systems of Ear Acupuncture. 4th ed. Edinburgh, London, New York, Oxford, Philadelphia, St. Louis, Sydney, Toronto: Churchill Livingstone/Elsevier; 2014.

18. Carpenter JS. The Hot Flash Related Daily Interference Scale: A tool for assessing the impact of hot flashes on quality of life following breast cancer. J Pain Symptom Manage. 2001;22(6): 979-989.

19. Chang P, Szymanski KM, Dunn RL, et al. Expanded prostate cancer index composite for clinical practice: Development and validation of a practical health related quality of life instrument for use in the routine clinical care of patients with prostate cancer. J Urol. 2011;186(3):865-872.

20. Lee KA. Self-reported sleep disturbances in employed women. Sleep. 1992;15(6):493-498.

21. Freedman RR. Physiology of hot flashes. Am J Hum Biol. 2001;13(4):453-464.
22. Hoikkala $H$, Haapalahti $P$, Viitasalo $M$, Väänänen $H$, Sovijärvi AR, Mikkola YO. Association between vasomotor hot flashes and heart rate variability in recently postmenopausal women. Menopause. 2010;17(2):315-320.

23. Freedman RR, Kruger ML, Wasson SL. Heart rate variability in menopausal hot flashes during sleep. Menopause. 2011; 18(8):897-900.

24. Thurston RC, Christie IC, Matthews KA. Hot flashes and cardiac vagal control during women's daily lives. Menopause. 2012;19(4):406-412.

25. de Zambotti M, Colrain IM, Sassoon SA, Nicholas CL, Trinder J, Baker FC. Vagal withdrawal during hot flashes occurring in undisturbed sleep: Hot flashes and autonomic activity. Menopause. 2013;20(11):1147-1153.

26. Frangos E, Ellrich J, Komisaruk BR. Non-invasive access to the vagus nerve central projections via electrical stimulation of the external ear: fMRI evidence in humans. Brain Stimul. 2015;8(3):624-636.

27. Arai YC, Sakakima Y, Kawanishi J, Nishihara M, Ito A, Tawada Y, Maruyama Y. Auricular acupuncture at the "shenmen" and "point zero" points induced parasympathetic activation. Evid Based Complement Alternat Med. 2013;2013: 945063.

28. Shi X, Litscher G, Wang H, et al. Continuous auricular electroacupuncture can significantly improve heart rate variability and clinical scores in patients with depression: First results from a transcontinental study. Evid Based Complement Alternat Med. 2013:2013:894096.

Address correspondence to: Tyvin Rich, MD, FACR Hampton University Proton Therapy Institute 40 Enterprise Parkway Hampton, VA 23666

E-mail: Tyvinr@gmail.com 